\title{
Les processus croisés de diffusion des innovations dans l'administration publique
}

\author{
Denis Harrisson \\ Université du Québec à Montréal
}

\section{Introduction}

1 es innovations connaissent généralement une période intense de création suivie d'une période de diffusion dans le milieu d'où elles proviennent. Plusieurs recherches ont porté sur le processus de création de l'innovation, alors que la diffusion reste encore marquée par des mythes et des difficultés de compréhension. Les théories diffusionnistes élaborées par Rogers et Abrahamson ${ }^{1}$ ont tendance à construire la diffusion comme un phénomène univoque qui repose sur les réseaux, sans trop tenir compte du type d'innovation devant être diffusé. Notre propos consiste donc à traiter de cette importante question et de construire le processus de diffusion en fonction du type et de la portée de l'innovation. L'approche de l'isomorphisme institutionnel révèle des éléments de compréhension de la diffusion fort pertinents aux organisations complexes qu'on retrouve dans l'administration publique. Notre démonstration repose sur trois innovations organisationnelles répertoriées dans l'administration publique québécoise que nous analysons quant

à la forme qu'emprunte la diffusion. Nous concluons en affirmant que plusieurs innovations sont nécessaires dans les organisations complexes, que les processus, bien qu'indépendants, s'entrecroisent et forment un système d'innovations organisationnelles. L'argumentation repose ici sur la théorie de l'isomorphisme organisationnel développée par Powell et DiMaggio ${ }^{2}$, ainsi que sur la théorie diffusionniste qui tente une explication des avancées de l'innovation et de ses ratés.

\section{L'innovation et l'administration publique}

Une innovation achevée prend la forme d'un ensemble de pratiques, de procédés ou de formes organisa- tionnelles qui connaissent du succès auprès des utilisateurs. En effet, une innovation demeure locale si elle ne peut s'étendre à d'autres milieux, au pire elle s'éteint. L'innovation est caractérisée par le processus, c'est-à-dire la conception et l'itinéraire d'un projet, d'une pratique ou d'une forme organisationnelle qui répond de manière adéquate, à la fois efficiente et légitime, à un problème ou à une difficulté. L'innovation est la création d'acteurs spécifiques en mesure de prendre des initiatives et intensément impliqués dans la résolution d'un problème. L'innovation est donc un processus social qui invite à l'interaction, à l'échange d'informations et de connaissances. La coopération entre des acteurs diversifiés et hétérogènes est un trait distinctif de l'innovation sociale ${ }^{3}$.

Les innovations dans le secteur public sont complexes. Elles naissent auprès d'organisations nombreuses que sont les ministères et les organismes publics, formant un ensemble cohérent dans la mesure où ces organisations possèdent une structure en tous points similaires parce que centralisée et organisée par le haut de la pyramide afin de maintenir les principes de l'équité et de l'égalité en matière de prestations de services comme en matière des relations entre les employés et l'employeur. Par ailleurs, ces organisations sont réparties sur un vaste territoire afin de livrer des services aux citoyens; elles possèdent donc une certaine marge de manœuvre qui permet à ses acteurs d'adapter les relations auprès des usagers. Le secteur public est vaste et comprend une variété de processus d'innovations, qu'ils soient technologiques, tel le gouvernement en ligne, ou organisationnels à travers la mise en place de nouveaux processus administratifs, ou encore développant de nouvelles façons de livrer les services. Ces innovations dépendent pour une bonne part des politiques publiques. Les proces- 
sus se produisent à différents niveaux impliquant plusieurs institutions et acteurs à la fois dans le secteur public de gouvernance nationale, régionale et locale. La particularité du service public est à l'effet qu'il n'est pas guidé par la rentabilité mais par l'offre de services et le développement de compétences des employés afin qu'ils puissent contribuer à l'amélioration du bien-être des collectivités.

Les administrations publiques ont été confrontées à de lourdes difficultés budgétaires au cours des vingt dernières années; elles subissent également les effets de la mondialisation. Le développement des politiques publiques est souvent interrompu par l'élection de gouvernements qui leur confèrent de nouvelles orientations en fonction de l'idéologie politique qui les anime. C'est pourquoi plusieurs réformes se sont succédées afin de réinventer les services gouvernementaux et les organisations qui les offrent ${ }^{4}$. Les organisations publiques sont difficiles à transformer. L'amélioration des performances n'est pas la seule mesure de l'efficacité gouvernementale. La conformité avec les règles administratives, les opinions des leaders publics, les pressions créées par des groupes d'intérêts multiples de même que les anticipations créées par les programmes sont inclus dans la performance gouvernementale. Comme le mentionnent Lenkowsky et Perry ${ }^{5}$ : «Le gouvernement est moins l'affaire de l'invention et de la réinvention que de l'évolution et du compromis ». Même avec une forte volonté et une implication sans faille de tous, il est difficile d'aller au-delà du cadre de l'action immédiate, et c'est bien l'un des problèmes des réformes centrales.

Le New Public Management (NPM) est une réforme qui introduit les pratiques de gestion du secteur privé orientées vers la promotion de l'efficience gouvernementale. Décrit tantôt comme une réforme, tantôt comme une idéologie, le NPM encourage fortement l'allègement des activités de l'État et initie pour ce faire différentes mesures innovatrices dans les structures comme le partenariat avec le secteur privé, la création d'agences, les réseaux avec le milieu associatif et communautaire, l'utilisation intense des NTIC (nouvelles technologies de l'information et de la communication), la mesure des performances de manière à améliorer la qualité des services aux citoyens, la décentralisation de l'autorité et la réduction de la taille de l'État, le tout suivi d'une réorganisation ${ }^{6}$. La réforme invite à des transformations à de multiples ni- veaux, d'une durée variable, impliquant plusieurs catégories d'acteurs. Il n'y a pas d'itinéraires proposés et plusieurs voies sont donc possibles, qu'elles soient centralisatrices, autoritaires et venant d'en haut ou alors qu'elles reposent sur la gouvernance partenariale, distributive et misant sur la diversification, la différenciation et le réseautage ${ }^{7}$.

\section{« Le gouvernement est moins l'affaire de l'invention et de la réinvention que de l'évolution et du compromis ».}

Les organisations publiques possèdent des structures denses difficiles à briser. Les réformes et autres innovations semblent partielles et insuffisamment diffusées. Certaines innovations prennent forme au niveau local, d'autres au niveau ministériel, d'autres encore au niveau national, mais rarement aux trois niveaux simultanément. Enfin, certains choix budgétaires et financiers de même que les orientations plus ou moins cohérentes et consistantes mises de l'avant par les directions et les choix politiques peuvent être contradictoires avec les intentions de transformer l'administration publique ${ }^{8}$.

Les processus sont complexes à la mesure de la composition d'organisations multiples et d'enjeux diversifiés du secteur. Pour ce faire, trois types de processus se sont développés : les mesures centralisées et obligatoires qui prennent la forme de lois ou de règlements; un processus de négociation qui transite par des accords entre les syndicats et l'État employeur; un processus émergent qui repose essentiellement sur des ententes entre les cadres de premier niveau et les employés qui travaillent dans des unités administratives spécifiques. Ces processus ne se croisent pas nécessairement, il n'y a pas toujours d'échanges organisés entre ces trois modes d'innovation. Par ailleurs, elles ne visent pas les mêmes cibles et l'on peut affirmer qu'il y a complémentarité bien qu'elles se développent sans connexion immédiate ${ }^{9}$. Chaque innovation est diffusée à travers un réseau qui lui est propre. Il y a plusieurs processus d'innovation simultanés. Avant de présenter ces innovations, voyons d'abord ce que rapporte la théorie diffusionniste.

\section{La théorie diffusionniste}

La théorie diffusionniste repose sur le principe de l'imitation d'une pratique innovante qui devient une 
norme sociale. Les innovateurs adoptent des comportements qui font école, car ce sont des personnes considérées dans leur milieu. L'innovation convient à leurs croyances. Ce sont des initiateurs qui essaient de rejoindre la majorité tardive, puis les retardataires. Les réseaux sociaux jouent un rôle fondamental dans la diffusion, mais cela fonctionne bien dans la mesure où la population est homogène. Que se passe-t-il quand il y a de grandes disparités entre les catégories d'acteurs dont l'hétérogénéité fonctionnelle et culturelle fragmente l'univocité des comportements et des résultats ? C'est la dynamique propre à l'administration publique.

\section{Lorsque les interactions sont fréquentes, les organisations peuvent imiter d'autres organisations qui sont proches géographiquement ou qui appartiennent à leur réseau de communication.}

La question qui intéresse Abrahamson est de savoir quand et par quel processus les innovations inefficaces sont diffusées, alors que des innovations efficientes sont rejetées. La réponse repose en partie sur les modes qui proposent des solutions simples à des problèmes complexes. Selon l'auteur, les innovations qui profitent aux organisations devraient être adoptées et les autres, au contraire, rejetées. Les innovateurs sont guidés par des objectifs d'efficacité. C'est l'approche du choix efficace. D'autres approches peuvent également être concurrentes, dont le processus d'imitation par effet de mode ou encore la sélection forcée. Ainsi, les organisations imitent celles qui font partie de leur groupe de transmission. Toutefois, il arrive que des organisations imitent celles qui ne sont pas de leur groupe lorsqu'un agent externe, un consultant par exemple, leur présente des connaissances qui leur conviennent. Lorsque les interactions sont fréquentes, les organisations peuvent imiter d'autres organisations qui sont proches géographiquement ou qui appartiennent à leur réseau de communication. Il y a alors un effet de contagion. Néanmoins une organisation dont les agents possèdent des attributs entièrement différents et qui n'est pas liée par le même réseau de communication est alors immunisée contre l'imitation et contre l'effet des communications de connaissances et de savoirs par les innovateurs. Mais est-ce bien toujours le cas? L'administration publique n'est pas liée au secteur privé, du moins les arran- gements institutionnels y sont-ils différents. Aussi, les organisations possèdent leurs propres processus d'innovation et de diffusion, en dépit du fait qu'elles sont influencées par la recherche d'efficacité. Pour bien comprendre, on se tourne alors vers une autre approche théorique, l'isomorphisme institutionnel.

Les travaux de Powell et DiMaggio sur l'isomorphisme n'ont pas pour but de traiter de l'innovation ni de sa diffusion. Ils tentent de comprendre comment les organisations adoptent des formes qui finissent pas se ressembler sous l'effet non pas de la recherche d'efficience, mais bien sous les pressions de leur environnement institutionnel. La diffusion des innovations emprunte des itinéraires semblables à l'isomorphisme organisationnel. En effet, lorsqu'une organisation développe une innovation et s'assure de sa diffusion, les organisations d'accueil discriminent alors les formes en fonction de ce qui se produit ailleurs dans leur environnement immédiat. Cette diffusion s'effectue alors sous trois formes distinctes que nous allons analyser en fonction de trois types d'innovation. Tout d'abord, l'isomorphisme coercitif intervient lorsqu'une organisation adopte des normes par obligation parce que les pressions exercées sont trop fortes. Les organisations se conforment à leurs obligations. Nous illustrons cette forme par les effets de la Loi sur l'administration publique. Ensuite, l'isomorphisme mimétique s'applique lorsque les organisations imitent ou copient intentionnellement les autres dans le but d'accroître leur légitimité. Ce type d'isomorphisme sera démontré à travers un cas d'innovation négociée que mettent de l'avant les Comités ministériels sur l'organisation du travail (CMOT). Enfin, l'isomorphisme normatif se déploie dans les organisations de manière indirecte par des normes et des valeurs importées par les personnes qui circulent d'une organisation à l'autre. Ce peut être l'embauche de nouveaux membres, des jeunes en particulier qui arrivent avec de nouvelles connaissances et de nouvelles façons de faire. C'est le cas qui s'applique bien à l'innovation émergente qui naît dans les organisations publiques.

Avec l'isomorphisme, l'organisation se complait sans avoir au préalable effectué une analyse de ce qu'elle devrait le mieux faire. La pression qui incite à imiter peut réduire le niveau d'innovation, car ce n'est plus la créativité qui est mise à l'honneur mais la simple adaptation de ce qui a été développé ailleurs. Ces formes de diffusion tendent à expliquer aussi pourquoi le milieu de l'administration publique est peu innova- 
teur. Il s'y prend peu de risques. Toutefois, il devient difficile pour les gestionnaires d'anticiper l'avenir et de reconnaître ou d'éviter les pressions externes qui menacent la survie de l'organisation. Ce sont ces facteurs qui expliquent le désir d'innover et de transformer l'organisation. L'organisation doit néanmoins devenir plus flexible et adaptée à son environnement externe. C'est ce que visent les trois innovations. Voyons maintenant comment l'isomorphisme se présente pour chacune des innovations retenues pour l'administration publique.

\section{Les innovations et leur diffusion}

A) Le premier cas de diffusion concerne l'isomorphisme coercitif. La Loi sur l'administration publique est une approche parallèle à d'autres approches innovatrices adoptées dans l'administration publique. C'est une approche que l'on peut qualifier d'innovation dogmatique au sens que Norbert Alter ${ }^{10}$ attribue à ce type d'innovation centralisatrice et autoritaire qui se déploie de haut en bas. La réforme de l'administration publique n'a guère d'équivalent dans d'autres secteurs d'activités économiques. Elle s'effectue par divers moyens, dont une loi qui devient la pierre angulaire des réformes autour desquelles se greffent les autres projets innovateurs. La réforme consiste à moderniser l'État, à modifier son rôle là où c'est nécessaire et à assurer aux citoyens des services de meilleure qualité et à des coûts réduits. Le processus est centralisé, il traverse l'ensemble des unités de l'administration publique sans exception. La diffusion se fait sur tout le territoire en respectant les principes d'égalité et d'universalité propres aux organismes publics. Les cadres responsables de l'application de cette loi sont assurés d'un meilleur suivi par les procédures administratives permettant l'acquisition de données fiables. Ces mesures concernent principalement la gestion par résultats et l'acquisition ou le perfectionnement des compétences et des qualifications.

De par sa nature, la loi acquiert ici un caractère obligatoire et coercitif. Dans ce cas précis, il n'y a ni participation, ni consultation, ni même échange d'informations auprès des principaux acteurs impliqués, si ce n'est les consultations habituelles précédant la législation. La loi se veut être un cadre de gestion concernant les pratiques, les procédures et les méthodes managériales; ce sont donc les gestionnaires de l'administration publique qui sont les principaux concernés. En pratique toutefois, il n'en est pas ainsi.
La loi n'a d'efficacité que si elle est validée par les acteurs qui devront la mettre en application. La loi introduit la gestion par résultats, la transparence et l'imputabilité de l'administration publique ainsi que l'obligation pour chaque ministère ou organisation publique d'adopter une déclaration de services aux citoyens quant au niveau de services offerts et un engagement à offrir des services de qualité. Différents outils de gestion devront alors être développés quant aux objectifs à atteindre et aux résultats qui seraient mesurables à l'aide d'indicateurs de performance, d'un plan de gestion des ressources humaines et de la relève, de la gestion des dépenses et de la reddition des comptes. Les effets de la loi sont sujets à débat. Certains acteurs se demandent comment une loi peut être bénéfique sans qu'elle ne soit approuvée et appropriée par les principaux artisans tels les superviseurs et les employés de même que les syndicats et associations professionnelles. Pour d'autres, les employés et les syndicats ont peu en commun avec les applications de cette réforme, aussi ils sont tenus à l'écart sauf pour des consultation ad hoc sur certains aspects de la loi qui les concernent, tel le plan de gestion des ressources humaines ou, dans certains cas, la déclaration des services au citoyen qui a été établie de concert avec les employés et les cadres et, dans des cas encore plus rares, avec les syndicats.

Cette innovation est introduite par obligation et maintenue par la force du cadre légal. Sa légitimité repose sur la loi et non sur des pratiques permettant une appropriation par les principaux acteurs. Ces derniers ne peuvent cependant pas développer d'autres innovations qui s'écarteraient des orientations et des pratiques qui découlent de ce cadre légal. Les innovations dogmatiques tiennent à distance les principaux intéressés, à l'exception évidente de ceux qui proposent un tel cadre pour transformer l'organisation. D'autant plus que les pratiques qui sont adoptées afin de transformer les modes de gestion dans l'administration publique sont empruntées au secteur privé et contraignent les gestionnaires à développer des pratiques qui soient mesurables au plan de leur efficience. Ainsi, les approximations et les incertitudes sont-elles effacées devant des mesures que l'on veut croire plus justes et plus efficaces, tout en respectant le contexte du service public. La loi s'applique, mais elle nécessite des innovations d'autre nature, moins coercitive.

Dans ce cas précis, l'innovation sera diffusée par les réseaux habituels prenant la forme de la structure 
hiérarchique, c'est l'isomorphisme coercitif. Le cadre légal n'est pas contre-productif, bien au contraire. La loi qui propose un cadre et qui initie des pratiques de gestion inédites pour ce milieu est voulue et même désirée par certains acteurs qui ne voient pas comment on peut transformer des organisations si intriqués les unes aux autres et si complexes avec des principes d'égalité, d'équité et d'universalité dans la prestation de services sans une proposition centralisatrice et obligatoire qui mène à la standardisation dans tous les lieux, petits et grands. Les cadres en sont les relais. Cette pièce législative prend ici le pas sur les autres mesures. C'est la pièce maîtresse de la réforme de l'administration.

B) L'isomorphisme normatif se déploie à travers des innovations qui proviennent d'initiatives adoptées par des acteurs locaux, les gestionnaires de premier niveau, les superviseurs et les employés. Le processus d'innovation est ici l'inverse du précédent et il acquiert un caractère volontaire par un réel souci d'appropriation par les acteurs de la base désireux de transformer les relations et d'assurer plus de transparence dans les échanges d'informations. Les innovations émergentes dans différentes unités sont ancrées dans la culture de ces unités; si elles pointent, c'est qu'elles sont recherchées et voulues par les acteurs. Elles participent à l'émergence des valeurs nouvelles propres aux nouvelles formes d'organisation du travail: participation, prise de la parole, adoption de méthodes de travail efficaces, travail en équipe, créativité et initiatives, nouvelles pratiques de gouvernance. Ces pratiques émergentes sont importantes bien qu'elles ne soient pas répertoriées. Elles ne relèvent que des initiatives prises par les acteurs locaux : superviseurs de premier niveau ou direction d'une unité de service et les employés. Elles desservent toutefois l'innovation de deux façons. D'abord, les acteurs qui s'y engagent développent les valeurs propres à l'innovation. Ils sont ouverts et créent un milieu de travail convivial dans lequel l'ensemble des acteurs tracent une voie par laquelle les objectifs de la nouvelle gestion publique, la recherche de performance, le rendement et l'amélioration du service au citoyen sont conjugués à la qualité de vie au travail, à la participation et à la prise de parole ainsi qu'aux normes de comportement recherché chez les fonctionnaires dans leurs interactions avec les citoyens et les usagers telles que l'intégrité, l'impartialité, la discrétion et l'éthique. Les acteurs se trouvent davantage prêts à faire face aux grandes innovations transversales pro- venant des instances centralisées de l'appareil administratif telles que la gestion par résultat. Ensuite, les pratiques émergentes de l'innovation participent de la création d'une culture d'innovation à travers laquelle les acteurs s'approprient des projets. Il devient alors plus simple de légitimer l'innovation, car elle peut plus rapidement être ancrée dans les attitudes et les valeurs de chacun sans procéder par une transformation de la structure de l'organisation du travail : mode de coordination, encadrement hiérarchique, segmentation des méthodes de travail.

Les pratiques émergentes d'innovation sont connues de tous les acteurs de l'administration publique bien qu'elles ne fassent pas l'objet d'une entente formelle. Ce sont des pratiques informelles évaluées comme étant certes capitales, mais non prioritaires. Dans la plupart des cas, elles sont laissées à elles-mêmes, et forcément ces pratiques se développent d'une façon asymétrique, très ancrées à certains endroits, très peu à d'autres.

La diffusion de l'innovation émergente n'est pas assurée car mal encadrée, mais elle est bien encastrée dans les lieux où elle est diffusée. Dans ce cas précis, les acteurs créent, ils inventent les formes organisationnelles dans lesquelles ils agissent, ils inventent les interactions, redistribuent l'influence et le pouvoir informel afin de créer des milieux de travail qui puissent répondre aux attentes et aux valeurs de ceux et celles qui les habitent. Mais ces innovations émergentes, bien que reconnues et distribuées de façon asymétrique dans les ministères et organisations publiques, sont diffusées de manière informelle par les principaux acteurs qui se gardent bien de rendre ces pratiques officielles. La diffusion emprunte alors l'itinéraire de l'isomorphisme normatif par la circulation des agents d'une unité à l'autre qui reprennent l'idée ou le projet dans un autre contexte organisationnel.

\section{Les pratiques émergentes de l'innovation participent de la création d'une culture d'innovation à travers laquelle les acteurs s'approprient des projets.}

Certaines valeurs et normes propres à ce type de pratique qui misent sur l'échange et le partage sont transmises d'une unité à l'autre parce qu'elles sont évaluées comme étant efficaces et légitimes. Ces pratiques se ressemblent bien qu'elles varient dans des ap- 
plications qui respectent le contexte local. Elles sont toutefois appropriées par les acteurs locaux qui développent alors des connaissances et des savoirs tacites transmissibles à travers la circulation des personnes d'un ministère ou d'un organisme à l'autre. Ces innovations locales ont un grand avenir dans l'administration. Ce sont des pratiques de proximité qui mettent en valeur les interactions de premier niveau et la confiance. La discussion porte alors sur la nécessité de mieux connaître et de mieux diffuser ces pratiques informelles, de les formaliser et de les ancrer dans un contexte élargi de la réforme de l'administration publique. Toutefois, ce type de diffusion a été bien limité. Les innovations émergentes ne sont pas diffusées partout, c'est une innovation asymétrique. C'est une forme d'isomorphisme normatif, car ce sont les cadres et les employés qui sont les porteurs de ces normes, règles et valeurs. Ils doivent circuler afin de diffuser ce type d'innovation.

C) Le troisième cas concerne l'isomorphisme mimétique. Le gouvernement du Québec, les syndicats, l'association des cadres et certaines associations professionnelles forment les parties prenantes d'une entente sur l'organisation du travail signée en 1995 et reconduite en 2004. Il s'agit du début d'un long itinéraire qui incite les fonctionnaires de l'administration publique à innover en matière d'organisation du travail. L'entente est un marqueur des relations entre les parties, car elle démontre la volonté de chacun à transformer l'organisation par des innovations et d'entreprendre une nouvelle phase de coopération en créant ce nouveau forum de discussion que représentent les Comités ministériels sur l'organisation du travail (CMOT) et le Comité sectoriel sur l'organisation du travail (CSOT). En matière d'organisation du travail, la question est associée à la réduction des dépenses budgétaires, à l'efficience en matière d'actions publiques et à l'amélioration de la qualité des services offerts aux citoyens et aux usagers des services publics. L'objet de discussion concerne le niveau d'emploi optimal, l'aménagement du temps de travail, la structure hiérarchique de l'administration publique et du rôle de chacun, la frontière des tâches, la classification des emplois, le cheminement de carrière, la sous-traitance. Les syndicats ont fait ajouter les questions relatives à la sécurité d'emploi et à la qualité de vie au travail. Les discussions qui ont été développées dans ces comités forment une part intégrale du processus d'innovation. L'innovation réelle n'est pas tant le document signé par l'ensemble des parties, mais bien les accords obtenus à la suite du dialogue entrepris dans cette structure de coopération. Ces accords sont avant tout pragmatiques, c'est-à-dire qu'ils relèvent de pratiques adoptées pour leur valeur heuristique en accord avec les expériences des acteurs en tant que sources de connaissance. Les acteurs ne désirent pas être prisonniers d'une définition conceptuelle ou encore d'une définition préétablie qui gène la progression d'une démarche collective en matière d'organisation du travail. C'est le travail concret qui donne un sens aux travaux des comités.

Les limites du dialogue, les sujets de discussion et le travail sur la représentation laissent place à une délimitation des travaux du comité par la pratique, l'expérience et le travail concret sur des dossiers spécifiques. C'est ainsi que les principales parties prenantes $\mathrm{y}$ trouvent leur compte. Les gestionnaires adhèrent à l'innovation que représentent les comités sur l'organisation du travail parce qu'ils peuvent légitimer leurs décisions directement auprès de leurs commettants. C'est une manière de partager l'information même si la responsabilité et l'imputabilité leur incombent en tout premier lieu. Les décisions seront mieux comprises par les parties impliquées et les malentendus pourront trouver une solution après avoir été analysés par tous. Pour les syndicats, l'innovation est une solution aux problèmes; ils participent à leur analyse, à leur diagnostic et à leur mise en œuvre. Les acteurs adoptent de nouveaux comportements dans les communications ouvertes, honnêtes sans abus d'autorité dans le cours des interactions. Les acteurs s'engagent dans de nouvelles relations qui développent leurs propres règles quant à l'égalité de statut, l'équivalence des arguments évoqués, l'absence de jugement, la conviction par la prise de parole et l'échange d'idées et des connaissances, l'absence de marchandage. C'est le produit d'un consensus entre ceux qui sont présents dans la relation. C'est une entité distincte qui se dissocie des arrangements habituels qui sont constitués.

L'innovation négociée n'est pas assurée du même suivi bien qu'elle soit le résultat d'une entente dans chaque ministère, à la suite d'un accord entre l'État et les syndicats. Les rencontres portent essentiellement sur l'organisation du travail. Mais l'entente doit être renégociée dans chaque ministère et organisme public de sorte que la diffusion est très inégale. Les rencontres qui ont lieu dans chaque ministère reposent sur la volonté des acteurs locaux de donner suite et d'y 
accorder de l'importance. Étant donné le processus négocié, les accords sont longs à obtenir L'isomorphisme est mimétique et on y recèle des tensions dans la dynamique. La diffusion des CMOT est réalisée par l'adoption des normes et des valeurs des autres organisations dans leur environnement immédiat. Les syndicats, les associations et les cadres supérieurs des différents ministères et organismes publics s'assurent de la similarité de structure.

\section{Conclusion}

Dans l'administration publique, trois démarches d'innovation se côtoient. Il y a d'abord une réforme de la gestion induite par la Loi sur la modernisation de l'administration publique; ensuite, la concertation patronale-syndicale à travers la mise en place des CMOT; et enfin, des actions volontaires conduites dans les ministères et portées par des acteurs locaux. Ces démarches sont autonomes tout en s'entrecroisant. Toutefois, certains préfèrent qu'elles soient parallèles et qu'il n'y ait pas de transversalité entre elles. Est-ce possible ? La réforme de la gestion, notamment la gestion par résultats, induite par la Loi sur l'administration publique, n'a pas d'équivalent dans le secteur privé. La réforme revêt donc un aspect coercitif et obligatoire qui se passe de toute démarche consultative auprès des syndicats. La loi est divisée en plusieurs parties et les modalités d'application dans les ministères ne peuvent faire l'économie d'un processus qui ne reçoit pas l'assentiment des employés et des cadres de premier niveau ainsi que des syndicats. Le CMOT devient alors un forum de discussion et d'échange sur le cadre de la loi et qui s'ajoute aux autres objets que sont les conditions de travail, la sécurité d'emploi, le coût des programmes proposés. Ces forums ne font pas l'unanimité parmi les acteurs qui œuvrent au sein des CMOT. Ils relèvent d'une pratique volontaire entre les acteurs et nul ne peut forcer le jeu. Plusieurs contraintes en retardent le démarrage : elles sont représentés comme étant inutiles par certains, ou encore marginalisés à la suite de la réforme légale de la gestion jugée prioritaire. D'autres croient que cette formalisation des comités n'ajoute rien à des pratiques présentes de manière informelle et elles se dédoublent.

Il s'agit bien sûr d'un idéal-type, car à chaque forme d'innovation correspond plus d'une façon de diffuser. Il s'agit ici d'identifier un mode dominant pour chaque type d'innovation, sachant que les deux autres s'entrecroisent. La loi est diffusée par obligation, mais les acteurs qui croient à son efficacité et à sa légitimité essaient de convaincre; en ce sens, la diffusion s'effectue également par normativité. Les CMOT sont imités d'un endroit à l'autre, alors que la circulation des cadres d'un ministère à l'autre en facilite l'adoption. De même, les innovations émergentes se développent par la circulation des membres de l'organisation, mais aussi par l'arrivée de nouveaux membres provenant de l'extérieur de l'organisation et y amenant de nouvelles idées et connaissances. Ces innovations sont aussi des imitations de ce qui se produit à l'extérieur. Par ailleurs, dans l'administration publique, il n'y a pas beaucoup d'embauche de personnes nouvelles et cela limite passablement les capacités de l'organisation d'innover par ces moyens.

\section{Il s'agit d'un idéal-type, car à chaque forme d'innovation correspond plus d'une façon de diffuser.}

Ces processus différents s'entrecroisent et viennent à former un système dans lequel chaque niveau d'innovation trouve une place légitime qui complète les autres. Le danger consiste alors à rendre prioritaires certains lieux d'innovation et à les placer en concurrence jusqu'à l'élimination des lieux moins intégrés. La loi et les processus centralisés possèderaient un avantage parce qu'ils sont balisés par des procédures fixes. Les autres lieux ne devraient cependant pas être négligés. En effet, on imagine mal une loi sans consultation préalable. De même, là où il n'y a pas d'obligation, il n'y a pas de diffusion partout, et là où il n'y a pas de mimétisme, il y a asymétrie des formes de participation davantage informelle qu'universelle.

Dans cet article, nous avons vu que les organisations complexes réparties sur un vaste territoire avec une mission commune, nécessitent plusieurs innovations auxquelles se greffent divers moyens de diffusion. Par ailleurs, afin de former un système d'innovation, un meilleur réseau de communication serait souhaitable, sinon les innovations évoluent en parallèle et peuvent se nuire, entrer en compétition et s'éliminer. Il peut arriver que le processus échoue à atteindre ses finalités, mais il réussit à prendre une nouvelle forme qui peut sembler légitime. La construction de sens n'est jamais atteinte, c'est ainsi que les organisations et leur gestion restent toujours dynamiques. 


\section{Notes et références}

1 Rogers, E., (1983). Diffusion of Innovations, New York, Free Press, $3^{\mathrm{e}}$ édition; Abrahamson, E. (1991). « Managerial Fads and Fashions: The Diffusion and Rejection of Innovations », Academy of Management Review, vol. 16, $\mathrm{n}^{\mathrm{o}} 3$, p. 586-612.

2 Powell, W.W. et P.J. DiMaggio (1991). The New Institutionalism in Organizational Analysis, Chicago, University of Chicago Press.

3 Alter, N. (2003). L'innovation ordinaire, Paris, PUF.

4 Kernaghan, K., B. Marson et S. Borins (2001). L'administration publique de l'avenir, monographies sur l'administration publique canadienne, $\mathrm{n}^{\circ} 24$, Toronto, Institut d'administration publique du Canada.

Lenkowsky, L. et J. Perry (2002). « Reinventing Government : The Case of National Service », Public Administration Review, vol. 60, nº 4, p. 298-307, 306.

6 Pollitt, C. et C. Bouckaert (2004). Public Management Reform : A Comparative Analysis, Oxford, Oxford University Press, $2^{\mathrm{e}}$ ed.; Thomas, P. (1996). « Visions versus Resources in Federal Program Review », dans A. Armit et J. Bourgault (eds), Hard Choices or no Choices, Toronto, Institut d'administration publique du Canada, p. 39-46; Peters, B.G. et D.J. Savoie (1998). Réformer le secteur pu- blic: où en sommes-nous?, Sainte-Foy, Presses de l'Université Laval; Borins, S. (2001). «Encouraging Innovations in the Public Sector », Journal of Intellectual Capital, vol. 2, no 3, p. 310-319; Roberts, A. (2001). « Altered States: Public Sector Restructuring and Governmental Capacity », dans R.P. Chaykowski (dir.), Globalization and the Canadian Economy: The Implications for Labour Markets, Society and the State, Kingston, School of Policy Studies, Queen's University, p. 105-130.

7 Bernier, L., M. Bouchard et B. Lévesque (2003). « La prise en compte de l'intérêt général par une économie plurielle », Téléscope, vol. 10, $\mathrm{n}^{\mathrm{o}} 2, \mathrm{p}$. 7-11.

8 Hood. C. et G. Peters (2004). « The Middle Aging of New Public Management : Into the Age of Paradox ? », Journal of Public Administration Research and Theory, vol. 14, $\mathrm{n}^{\mathrm{o}} 3$, p. 267-282.

9 Harrisson, D., N. Laplante et G. Bellemare (2006). « Innovations du travail et syndicats de la fonction publique : un partenariat à construire », Annals of Public and Cooperative Economics, vol. 77, no 2, p. 167-195.

10 Norbert Alter présente plutôt l'invention dogmatique, l'innovation dogmatique étant notre expression. Pour Alter, une invention dogmatique ne se rend pas au stade de l'innovation, car elle n'est jamais emparée par le corps social dans lequel elle circule. Voir Alter 2003, op. cit. 